EXEMPLARIa Classica

Journal of Classical Philology

23, 2019, $\mathrm{xx}-\mathrm{xx}$

ISSN 1699-3225

\title{
AL CLASSICISTA CHE VERRÀ. IN MARGINE AD UN RECENTE MANUALE DI FILOLOGIA CLASSICA ${ }^{1}$
}

\author{
Maria Chiara Scappaticcio \\ Università degli Studi di Napoli ‘Federico II' - ERC-Project PLATINUM \\ mariachiara.scappaticcio@unina.it.
}

Nel 2019 I classici dal papiro a Internet di Fabio Stok è alla settima ristampa dal 2012, nel 2017 usciva in lingua inglese e tedesca² ${ }^{2}$ questi dati e l'essenza del volume racchiusa nel pur breve titolo sono abbastanza per capire a primo colpo che ci si trova dinanzi ad un lavoro importante, decisamente attuale, destinato ad un 'futuro' che non è soltanto quello del successo editoriale ma anche (e soprattutto) quello della formazione della più giovane generazione di classicisti - che, in questo caso, equivale a dire di filologi classici. Si tratta, dunque, sì, di una 'storia della filologia classica' - con quanto di più complesso e poliedrico questa possa contenere - ma è soprattutto un progetto formativo che esce tanto più ampliato e rafforzato dalla versione inglese, lontana dall'esserne semplicemente una traduzione: Classics from papyrus to the internet vede ampliare il titolo con un sottotitolo che ne sintetizza la volontà di introdurre alla trasmissione e alla ricezione dei classici, vede affiancarsi a Fabio Stok i due statunitensi Jeffrey M. Hunt e R. Alden Smith, vede la creazione di un'appendice costituita da un sito web che

* ${ }^{1}$ Questa ricerca si inserisce nel quadro del progetto PLATINUM (Papyri and Latin Texts. Insights and Updated Methodologies. Towards a Philological, Literary and Historical Approach to Latin Papyri), finanziato dallo European Research Council (ERC-StG 2014 n636983) e di cui sono la responsabile scientifica e Principal Investigator.

Jeffrey M. Hunt, R. Alden Smith and Fabio Stok. Classics from Papyrus to the Internet: An Introduction to Transmission and Reception. Ashley and Peter Larkin Series in Greek and Roman Culture. Austin: University of Texas Press, 2017, xi+344 pp., \$ 29.95 (pb). ISBN 978-1-4773-1302-2

${ }^{2}$ F. Stok, I classici dal papiro a Internet, Roma; il volume è uscito come numero 807 nella collana Studi Superiori dell'editore Carocci. Per la traduzione tedesca si veda: Vom Papyrus zum Internet: eine Geschichte der Überlieferung und Rezeption der antiken Klassiker. Übersetzung von Christiane Reitz in Zusammenarbeit mit Torben Behm, Markus Ersten und Lars Keßler, Rahden 2017. 
completa il volume ma che risulta maggiormente dinamico nella misura in cui sia progressivamente aggiornato ed arricchito dagli autori ${ }^{3}$.

La finalità di questo nuovo progetto editoriale gemmato su quello già affermato di Stok è duplice e chiaramente delineata nelle pagine prefatorie (IX-XI): tracciare la storia degli studi classici e parlare ad un pubblico in formazione, alla nuova generazione di classicisti, che vedrà offerti strumenti complementari e aggiornati rispetto a quelli di due degli illustri precedenti menzionati dai tre autori, i precedenti di Leighton D. Reynolds e Nigel G. Wilson ${ }^{4}$, da un lato, e di Rudolf Pfeiffer ${ }^{5}$, dall'altro, autori di lavori che, nonostante la prospettiva differente, rappresentano un punto di riferimento necessario per i primi quattro capitoli del volume. Nel sesto capitolo, la 'lingua' cambia e diventa ulteriormente familiare al lettore cui il volume è destinato, e questo per ragioni semplicemente legate al tempo: Pfeiffer non poteva avere a disposizione uno strumento come l'Ibycus' creato da David Packard nel 1985 e, di lì, la versione online del TLG (attiva dal 2001), né Reynolds e Wilson hanno potuto registrare una banca dati tanto preziosa come il 'Digital Scriptorium'; eppure, strumenti come questi hanno arricchito il panorama degli studi classici e di questi beneficia il lettore di Classics from papyrus to the internet.

Il Foreword (1-4) di Craig W. Kallendorf, d'altro canto, insiste sulla necessità di capire e contribuire ai cambiamenti della filologia classica come disciplina - come critica testuale, come storia degli studi, come tradizione classica - e sull'importanza del concetto jaussiano di ricezione dei classici. La ricezione costituisce uno dei fils-rouges che attraversano il volume, a corroborare il quale potrà, in futuro, contribuire una rinnovata riflessione sulla circolazione dei testi che - per molti versi complementare alla ricezione stessa - permette di meglio tratteggiarne i milieux cui le opere vennero destinate ed in cui vennero utilizzate anche da una prospettiva squisitamente geografica che lascia più chiaramente intravedere le sfumature di una civiltà letteraria.

Il primo capitolo - Writing and Literature in Antiquity (5-45) - ̀̀ una sintesi sulla storia della letteratura antica, intesa come storia della scrittura e delle scritture: i temi trattati sono molti, e vanno dalla nascita e dall'affermazione dell'alfabeto al passaggio - graduale passaggio, e, per qualche tempo, convivenza - dal rotolo al codice di papiro e di pergamena. Epigrafia, papirologia, education e literacy sono soltanto alcuni tra i concetti chiave che ne escono illuminati. L'attenzione a tutti i supporti scrittori, dalle iscrizioni alle tavolette - passando dall'area pompeiana di Cecilio Giocondo

${ }^{3} \mathrm{https} / /$ sites.baylor.edu/papyrustointernet/.

${ }^{4}$ L.D. Reynolds, N.G. Wilson, Scribes and Scholars: A Guide to the Transmission of Greek and Latin Literature, Oxford 1968.

${ }^{5}$ R. Pfeiffer, History of Classical Scholarship, 1300-1850, Oxford 1976. 
alla britannica Vindolanda -, è marcata e rimarchevole, al pari della chiara illustrazione di 'strumenti di lavoro' - come il CIL o la cosiddetta 'convenzione di Leida' - necessari per settori della ricerca settoriale di epigrafia e papirologia e al pari delle sintesi relative alle antiche biblioteche e al commercio librario. Né manca l'enfatizzazione della difficoltà di circoscrivere categoricamente i limiti della ricerca in settori 'marginali'. Basti qui l'esempio relativo alla papirologia, a definire la quale viene precisato, prima, che leggere, descrivere e pubblicare i frammenti superstiti sia compito del 'papirologo's e, poi, che l'interpretazione dei papiri 'letterari' è piuttosto dominio di filologi e studiosi della letteratura ${ }^{7}$ : se, però, l'interpretazione dei papiri letterari da parte di filologi e studiosi della letteratura è fondata su edizioni papirologiche non aggiornate, scarsamente affidabili o persino non fondate sull'esame diretto dei testimoni papiracei non ha i presupposti per essere essa stessa un'esegesi affidabile ${ }^{8}$, e c'è da chiedersi se l'acquisizione di competenze di base come quelle paleografiche siano divenute semplicemente indispensabili per un 'filologo classico' che voglia capire e criticamente esaminare la tradizione di un testo e la sua storia attraverso i testimoni più e meno antichi. D'altro canto, l'attenzione dei tre autori per la storia della scrittura e delle sue forme - insomma, per la paleografia - è viva ed emerge distinta in tutti i capitoli del volume.

Gli sviluppi più antichi della 'critica testuale' vengono ripercorsi nel secondo capitolo - Grammar, Scholarship, and Scribal Practice from Antiquity to the Middle Ages (46-84) -, dalla scriptio continua e dai segni di punteggiatura - e, spesso, di 'lettura' a voce alta - alla scoliastica, dalla costituzione di un codice e delle sue possibili scritture alla confezione in scriptoria e ai possibili errori di copia. Gli aspetti della produzione e della circolazione libraria, soprattutto nella Tarda Antichità ma fino al Medioevo, si trovano ulteriormente illustrati all'interno del terzo capitolo - Classical Reception from Antiquity to the Middle Ages (85-148). Si tratta, d'altro canto, di questioni complementari e fondanti, ad illuminare le quali contribuisce

- Si veda 24: «it falls to the papyrologist to read, describe, and publish extant fragments».

$7 \mathrm{Si}$ veda 24: "the interpretation of literary papyri falls to philologists and literary scholars, since those texts are part of a literary tradition at the center of the field of philology».

${ }^{8}$ Per quanto concerne i testi latini su papiro questo tipo di difficoltà ed una nuova metodologia di ricerca è tratteggiata in M.C. Scappaticcio, Papyri and LAtin Texts: INsights and Updated Methdologies. Towards a philological, literary, and historical approach to Latin papyri (PLATINUM Project ERC-StG 2014 no. 636983), in A. Nodar, S. Torallas Tovar (eds), Proceedings of the 28th Congress of Papyrology (Barcelona 1-6 August 2016), Barcelona 2019, 619-27. 
ora in modo determinante il più recente e capitale volume di James Zetzel ${ }^{9}$. La prospettiva degli autori è essenzialmente orientata sull'Occidente, ma all'inquadramento sul 'Greco in Occidente' (107-113) i rinnovati studi sulla circolazione del 'Latino in Oriente' offriranno un ampliamento non secondario tanto più se si riflette sulla fortuna di strumenti formativi di possibile origine antica ed orientale come i cosiddetti Hermeneumata Pseudodositheana nell'Occidente medievale, dove vennero adeguati a esigenze nuove che hanno contribuito a determinarne un'interessante e complessa stratificazione testuale ${ }^{10}$.

Dall'Umanesimo la storia della filologia si spinge fino alla nascita dell'Altertumswissenschaft all'interno del quarto e del quinto capitolo, con la nascita della stampa come spartiacque cronologico e con l'enfatizzazione di come la storia della filologia coincida, per molti versi, con la storia della cultura e rifletta il corso della storia stessa ${ }^{11}$. E la parabola della storia viene seguita fino ad arrivare ai Tools for the Modern Scholar (221-240)12: il sesto (innovativo) capitolo è un'illustrazione chiara ed esaustiva - benché necessariamente selettiva - delle risorse necessarie - cartacee e digitali - per chi, oggi, affronti lo studio della filologia classica. Si tratta senz'altro del terreno più 'mobile' per il fatto che è in continuo sviluppo e al cui aggiornamento -

9 J. Zetzel, Critics, Compilers, and Commentators: An Introduction to Roman Philology, 200 BCE-800 CE, Oxford 2018.

${ }^{10}$ Quanto al 'Latino in Egitto' la panoramica più esaustiva e chiara cui si rinvia per ulteriori approfondimenti e riferimenti bibliografici è quella di J.N. Adams, Bilingualism and the Latin Language, Cambridge 2003, 527-641; nuovi studi sono in cantiere nell'ambito del progetto PLATINUM con la finalità di stimolare una rinnovata riflessione sulla circolazione della lingua e della letteratura latina in Oriente anche attraverso la realizzazione di un Corpus of Latin Texts on Papyrus (Cambridge University Press). In tempi recenti ulteriori impulsi sono stati dati alla ricerca sul bilinguismo greco-latino e latino-greco - si veda, ad esempio, la summa di F. Biville, Le bilinguismo gréco-latin, «Lalies» 37, 2017, 45-105 - e sui cosiddetti Hermeneumata Pseudodositheana e sui glossari bilingui, a proposito dei quali ci si limita a rinviare agli importanti lavori di Eleanor Dickey - tra cui le due edizioni dei colloquia degli Hermeneumata, nell'introduzione ai quali viene tracciato un'utile ed esaustivo quadro della storia della tradizione di questa specifica e peculiare categoria di testi; si veda E. Dickey, The Colloquia of the Hermeneumata Pseudodositheana I-II, Cambridge 2012-2015.

${ }^{11} \mathrm{Si}$ vedano rispettivamente il quarto capitolo, Classics and Humanists (149-190), ed il quinto, Classical Texts in the Age of Printing (191-220).

${ }^{12}$ Il volume è chiuso dalle note (241-299) - la cui consultazione nella sezione conclusiva del volume è poco pratica -, da un'esaustiva ed utile bibliografia (301$324)$ e da un indice (325-344). Va, inoltre, sottolineato che il volume è corredato di numerose illustrazioni, preziose e funzionali a chiarire ulteriormente i problemi illustrati. 
come si è detto - provvede la pagina web complementare al volume stesso. Questa 'mobilità' è tanto più evidente se si riflette sul fatto che la creazione del Digital Corpus of Literary Papyri (DCLP) è successiva alla pubblicazione del volume ${ }^{13}$, così come lo è il prezioso Open Access ai volumi del Thesaurus Linguae Latinae (TLL, o ThLL $)^{14}$, e ancora lo saranno nuovi ed importanti progetti - molti in divenire e da rendere fruibili, molti ancora da costruire perché la nostra disciplina resti in vita e ne viva di nuova.

${ }^{13}$ Il $D C L P$ (http://www.litpap.info), pertanto, non si trova menzionato né nel volume né, allo stato attuale (agosto 2019), sul sito web.

${ }^{14}$ http:/ / www.thesaurus.badw.de/tll-digital/tll-open-access.html 
